\title{
Waterproof and moisture permeability performance of laminated fabrics with electrospun nano fibrous membrane
}

\begin{abstract}
A waterproof and moisture permeable fabric was fabricated by laminating polyester (PET) or polyurethane (PU) electrospun nano fibrous membranes inside of common blended fabrics, and the obtained laminated fabrics had good waterproof and moisture permeable performance. The waterproof and water permeable performance of the laminated fabrics were dependent on the component and thickness of the electrospun nano fibrous membranes that laminated inside by hot pressing. The laminated fabric with PET/PU composite electrospun nano fibrous membrane as functional layer has excellent moisture permeability of up to $8.82 \mathrm{~kg} /\left(\mathrm{m}^{2} \bullet \mathrm{d}\right)$, air resistance value of $6.2 \mathrm{kPa} /(\mathrm{s} \bullet \mathrm{m})$ and hydrostatic pressure of $36.1 \mathrm{~cm} \mathrm{H}_{2} \mathrm{O}$, respectively. Moreover, the hydrostatic resistance of the laminated fabric combined with PU electrospun nano fibrous membranes is higher than that of the laminated fabric combined with PET nano fibrous membranes and has a better hand feeling as well.
\end{abstract}

Keywords: electrospun nano fibrous membranes, laminated fabric, breathability, moisture permeability, hydrostatic pressure
Volume 2 Issue 3 - 2017

\author{
Bin Wang', Jiaona Wang,' Shuyu Zhao,' \\ Zefang Yin, ' Congju $\mathrm{Li}^{2}$ \\ 'School of Materials Science \& Engineering, Beijing Institute of \\ Fashion Technology, China \\ ${ }^{2}$ Beijing Institute of Nano energy and Nano systems, China
}

\begin{abstract}
Correspondence: Congju Li, Beijing Institute of Nano energy and Nano systems, Chinese Academy of Sciences, 30 Xueyuan Rd, Haidian, Beijing 100083, China, Tel 86-10-82854885, Email licongju@binn.cas.cn

Jiaona Wang, School of Materials Science \& Engineering, Beijing Key Laboratory of Clothing Materials R \&D and Assessment. Beijing Institute of Fashion Technology, Beijing 100029, China, Email wangjiaona_20II@sina.cn
\end{abstract}

Received: June 26, 2017 | Published: July 17, 2017
Abbreviations: PET, polyester; PU, poly urethane; DMF, N, n-dimethylformamide; TFA, trifluoroacetic Acid; DMC, dichloromethane; COPA, copolyamide

\section{Introduction}

Waterproof and moisture permeable fabrics, also known as waterproof and breathable fabrics, are widely used in our daily life. This permeability to water vapor but resistance to water droplets are not only applied in textile industry but also can be employed in medical applications, membrane distillation, moisture regulation of buildings, and in humidification for the conservation of art and paper. ${ }^{1-4}$ They can be divided into high density woven fabrics, coated fabrics and laminated fabrics. The laminated fabric could display the excellent properties of all the component fabrics, and its multilayered structure is independent of each other and has good combination. Simultaneously, the processing technique of laminated fabric also avoids solvent pollution, labor protection and fire protection and other issues.

In recent years, nano fibers prepared by electro spinning technology have been used in filtration, ${ }^{5}$ sensors,${ }^{6}$ tissue engineering ${ }^{7}$ and protective clothing. ${ }^{8}$ Electrospun nano fibrous membranes possess unique fabric construction, such as ideal porous structure with small pores, fine fiber diameter, large surface area and light weight, thus becoming the first choice for the preparation of multifunction and high-performance clothing fabrics. ${ }^{9}$ And the pore size of the electrospun nano fibrous membranes can be designed to be much smaller than the diameter of the smallest water droplets $(2 \mu \mathrm{m})$ and much larger than the diameter of the water vapor molecules $(0.3 \sim 0.4 \mu \mathrm{m})$. In addition, when laminated fabrics consisting of electrospun nano fibrous membranes it would be endowed good water vapor transmission capability due to the porous structure of the electrospun nano fibrous membranes. Based on the unique breathable characteristic, this laminated fabric is promising to construct protective clothing that the evaporation cooling process occurs in the pores of electrospun nano fibrous membranes. ${ }^{10-12}$ However, there are few reports on how to use electrospun nano fibrous membranes as functional layers for waterproof and moisture permeable laminated fabrics. In this study, polyester (PET) and polyurethane (PU) were directly spun on hot melt adhesive, and then they were hot pressed with fabric and lining to obtain laminated fabrics and its water resistance and vapor permeability were studied.

\section{Experiments}

\section{Materials}

In this work, Nylon and cotton blended fabrics were used as shell fabric and warp knitted fabric was used as lining. Polyester (PET) and polyurethane (PU) were purchased from the Far Eastern Industry (Shanghai) Ltd., N, N-dimethylformamide (DMF), trifluoroacetic acid (TFA) and dichloromethane (DMC) are all analytical grade and were purchased from Beijing Tonguing Fine Chemicals Co., Ltd., copolyamide (COPA) was used as hot-melt adhesives and purchased from Tangshan Fenghe Plastic Co., Ltd.. Electro spinning device was laboratory made.

\section{Experimental process}

Preparation of electrospun nano fibrous membranes: Preparation of PET nano fibrous membranes: In this procedure, PET particles were dissolved in DMF and TFA (the volume ratio is 1:2) to form a $20 \%$ (by mass fraction, the same below) electro spinning solution. During the electro spinning process, the hot melt was coated on the receiver first, and then a high voltage of $20 \mathrm{kV}$ was applied to electro spin PET solution. The distance between the spinning nozzle and the receiver was $18 \mathrm{~cm}$, the humidity was in the range of $10 \% \sim 15 \%$ 
and the receiver rotation speed was $70 \mathrm{r} / \mathrm{min}$. Finally, PET nano fibrous membranes were obtained with different spinning time $(4 \mathrm{~h}, 6 \mathrm{~h}$, 8h, 10h and 12h), which were noted as PET 4, PET 6, PET 8, PET 10 and PET 12, respectively. The PU nano fibrous membranes were fabricated through a similar process. In this procedure, PU particles were dissolved in DMF to form 6\% homogeneous electro spinning solution. The spinning voltage was $18 \mathrm{kV}$, and the other conditions were the same as that of PET. Then PU nano fibrous membrane was obtained by spinning for $10 \mathrm{~h}$.

Preparation of laminated fabrics and determination of optimum hot pressing conditions: Copolyamide (COPA) was used as hot-melt adhesive during all the following hot pressing experiments. Firstly, the lining (sizing face upwards), and electros spun nano fibrous membrane, the shell fabric (sizing face downwards) were layered in turn on the hot machine. Then the different hot pressing time $(15 \mathrm{~s}, 17.5 \mathrm{~s}, 20 \mathrm{~s}$, $22.5 \mathrm{~s}$ and $25 \mathrm{~s})$ and hot pressing temperature $\left(100^{\circ} \mathrm{C}, 110^{\circ} \mathrm{C}, 120^{\circ} \mathrm{C}\right.$, $130^{\circ} \mathrm{C}$ and $140^{\circ} \mathrm{C}$ ) were set. The schematic diagram of hot pressing process for laminated fabrics is shown in Figure 1.
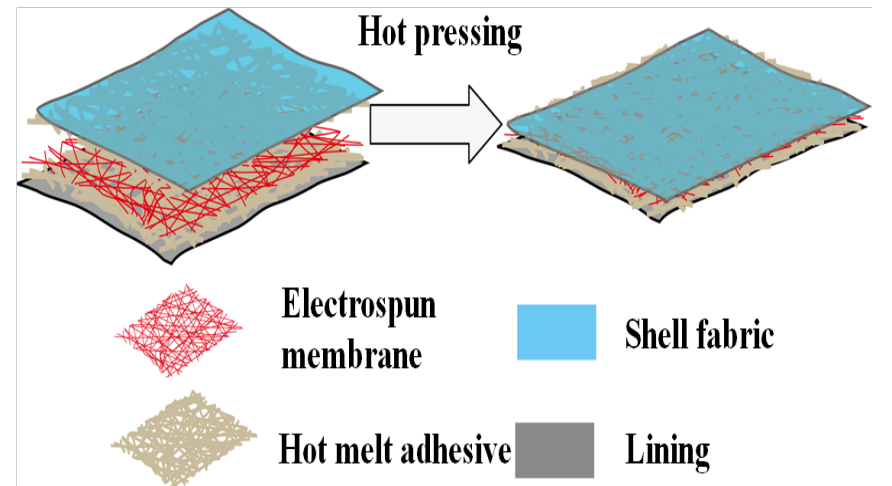

Figure I The schematic diagram of hot pressing process for laminated fabrics.

Performance testing of laminated fabrics: The morphology of COPA and electro spun nano fibrous membranes were characterized by scanning electron microscope (JSM-6360LV, Japan Electronics Co., Ltd.). The fiber diameter was gauged employing a custom code image analysis program (Smile View). Contact angle measurement: The sample was placed on a test bench of a video optical contact angle tester (OCA20, Data physics, Germany), the deionized water in the capillary was pushed at a rate of $2 \mu \mathrm{L} / \mathrm{s}$, and the image was frozen and the contact angle of the sample was analyzed by SCA20 software.

Air permeability (air resistance) test: Using an air resistance tester (KES-F8-AP1, KATO Company), the air resistance values of laminated fabrics and electrospun nano fibrous membranes were tested under a constant condition (humidity of $65 \%$ and temperature of $\left.20^{\circ} \mathrm{C}\right)$ for $24 \mathrm{~h}$. The air resistance value $[\mathrm{kPa} /(\mathrm{s} \cdot \mathrm{m})]$ was taken the average of ten sampling point.

In order to determine the optimum hot pressing conditions, the peel strength of the laminated fabric was then measured by referring to criterion (FZ/T01010-1991) of "Coated fabrics-Determination method of coating adhesion". A Fabric strength instrument (YGB026E-500, Wenzhou Dahong Textile Instrument Co., Ltd.) was used to test the peel strength of the laminated fabrics prepared by hot pressing. The size of test sample was fixed at $2.5 \mathrm{~cm} \times 20 \mathrm{~cm}$. The tensile force and tensile distance was $300 \mathrm{~N}$ and $10 \mathrm{~cm}$, respectively.

Waterproof and moisture permeability test: According to the criterion (GB/T4744-1997) of "Determination of hydrostatic resistance of impermeability of textile fabrics", the hydrostatic pressure of laminated fabric was test under the following conditions using resistant hydrostatic pressure tester (SDLATLAS M018 Hydrostatic Head Tester, US SDL Atlas Ltd.). The water temperature was $(20 \pm 2)^{\circ} \mathrm{C}$, the increase rate of water pressure was $10 \mathrm{~cm} \mathrm{H}_{2} \mathrm{O} /$ min and the maximum water pressure was added up to $3000 \mathrm{~cm} \mathrm{H}_{2} \mathrm{O}$. According to the criterion (GB/T12704.12009) of "Textile fabric moisture permeability test method Part 1: Moisture method", the breathability of laminated fabrics were tested using moisture test device (DH-400, Japan Daejeon Scientific Institute of Fine Materials). During the testing, the air temperature was controlled at $(38 \pm 2)^{\circ} \mathrm{C}$, the relative humidity was controlled at $(90 \pm 2) \%$ and the fine-grained anhydrous calcium chloride was used as the desiccant.

Water absorption test: The sample was dried first at $38.5^{\circ} \mathrm{C}$ to get a constant weight, noted as $\mathrm{W}_{0}(\mathrm{~g})$. Then immersed the samples in deionized water for $1 \mathrm{~h}$, took out the fabric and absorbed the surface water droplets with filter papers (the whole process should take no more than $1 \mathrm{~min}$ ), weighted the fabric and recorded as $\mathrm{W}_{1}(\mathrm{~g})$. The water adsorption capacity of fabric was calculated by the following equation:

$$
\text { Water absorption }(\%)=\left(W_{1}-W_{0}\right) / W_{0} \times 100 \%
$$

Warmth retention of laminated fabrics was tested by KES-F7II(Japan KATO TECH Co., Ltd.). Firstly, cutting the fabric into size of $20 \mathrm{~cm} \times 20 \mathrm{~cm}$ and keeping in a constant condition (humidity of $65 \%$ and temperature of $20^{\circ} \mathrm{C}$ ) for $24 \mathrm{~h}$. Then, the warmth loss value was tested with and without fabric, respectively. Finally, the warmth retention value was calculated by the testing system.

\section{Results and discussion}

\section{Effect of hot pressing on peel strength}

According to the similarity and inter miscibility, the solubility parameter of adhesive and electrospun membrane should be similar so that the peel strength would be as high as possible. Thus, the choice of adhesive is of great importance, as well as the sizing method. In this work we use a mesh-shaped COPA hot-melt adhesive to bond electrospun membranes with lining and shell fabric, the hot pressing structure as shown in Figure 1. We put COPA both on the upper side and lower side of electrospun membrane first and then hot pressing. The morphology of COPA we used is shown in Figure 2. This adhesive is of fibrous morphology with the average diameter of about $30 \mu \mathrm{m}$. In the practical application, the adhesive is required to have low melt viscosity and good mobility due to the fast hot pressing process. Furthermore, the mobility should not be too strong, otherwise the melt adhesive would penetrate through the fibrous membranes and/ or fabrics.

Beside the choice of adhesive and sizing method, the hot pressing condition is vital for the peel strength of laminated fabrics. In order to decide the hot pressing condition, the COPA was first analyzed by DSC, as displayed in Figure 3. The melting temperature is not obvious and set various hot pressing temperatures at $100^{\circ} \mathrm{C}, 110^{\circ} \mathrm{C}, 120^{\circ} \mathrm{C}$, $130^{\circ} \mathrm{C}$ and $140^{\circ} \mathrm{C}$. In the other hand, the hot pressing time is adjusted at $15 \mathrm{~s}, 17.5 \mathrm{~s}, 20 \mathrm{~s}, 22.5 \mathrm{~s}$ and $25 \mathrm{~s}$, respectively. The peel strength (adhesion strength) of laminated fabric is mainly related to the hot pressing condition. Generally, the stronger the adhesion is, the larger 
the peel strength is. By using PET 8 as a sample, a series of laminated fabrics were fabricated and the histogram of peel strength at different hot pressing conditions was illustrated in Figure 4. As shown in Figure 4 , the peel strength of laminated fabrics are all larger than $5 \mathrm{~N} / 2.5 \mathrm{~cm}$ when the hot pressing temperature within $120 \sim 130^{\circ} \mathrm{C}$ and hot pressing time within 17.5 22.5s. Further, the peel strength reached up to 8.34 $\mathrm{N} / 2.5 \mathrm{~cm}$ at hot pressing condition of $130^{\circ} \mathrm{C}$ and $17.5 \mathrm{~s}$. The best hot pressing conditions for the laminated fabric consisting of other PET electrospun membranes and PU electrospun membrane are the same as that of PET8 electrospun membrane.

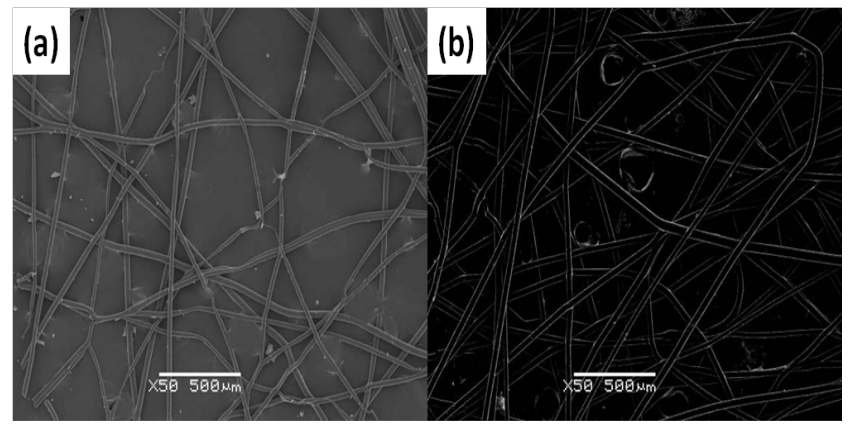

Figure 2 The SEM morphology of mesh-shaped COPA hot-melt adhesive.

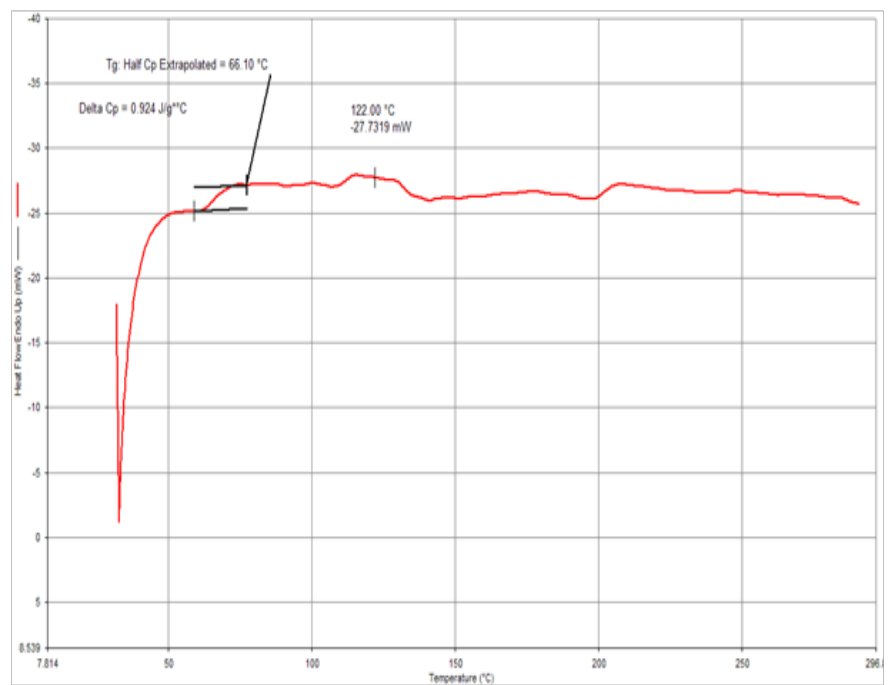

Figure 3 The DCS curve of COPA.

\section{Electrospun membranes properties}

Thickness and surface density: The thickness and surface density of PET and PU electrospun nano fibrous membranes were calculated by the formulas (2) and (3) and presented in Table 1.

$$
\begin{aligned}
& \operatorname{Porosity}(\%)=\left(1-\rho_{\mathrm{e}-\text { spun }} / \rho_{\text {powder }}\right) \times 100 \% \\
& \rho_{\text {e-spun }}=m / V=m /(l \times d \times h)
\end{aligned}
$$

Where $m$ is the mass of the membrane, $l$ is the length of the membrane, $d$ is the width of the membrane, $h$ is the thickness of the membrane, $\rho_{\text {powder }}$ is the density of raw materials (polymer particle).

It can be seen from Table 1 that the electrospun nano fibrous membranes, PET 6 and PET 8, with the same material but different spinning time have similar porosity, fiber diameter and water contact angle. And the thickness and surface density are increased with the increasing spinning time. However, comparing with PET membranes the fiber diameter of $\mathrm{PU}$ is much smaller, so the thickness and surface density are lower than that of PET 8 membranes even with a longer spinning time. It is notable to say that all the samples have high porosity, which is benefit for the air and water vapor penetrate the membrane layer. In addition to the high porosity, the water contact angle of electrospun nano fibrous membranes as shown in Figure 5 are all larger than $90^{\circ}$, so that the water proofness may size up the requirement of protective clothing. We can summarize from Table 1 that the characteristics of electrospun nano fibrous membrane are dependent on both the raw material property and spinning condition.

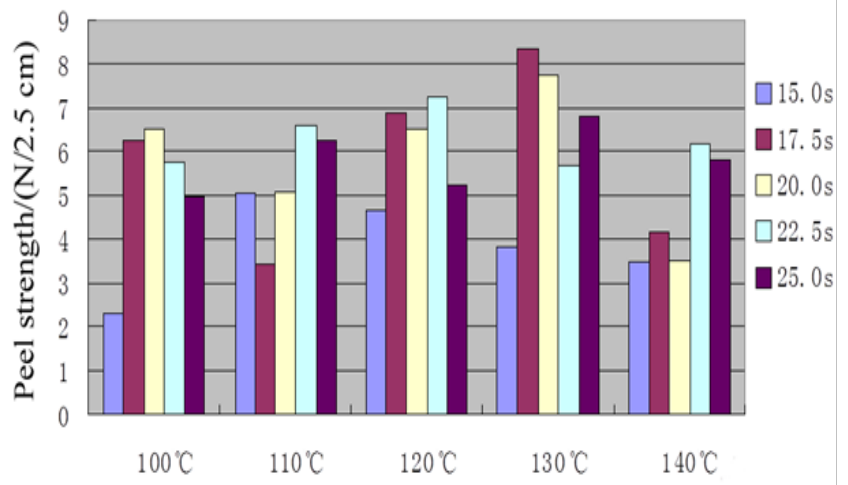

Figure 4 Effect of hot pressing time and temperature on the peel strength of laminated fabric with PET8 electrospun membrane.

Membrane morphology: The morphology of PET and PU nano fibrous membrane are show in Figure 5. PET and PU nano fibers were smooth and stacked randomly. The fiber diameters of PET and PU are mainly in the range of $800 \sim 900 \mathrm{~nm}$ and $300 \sim 400 \mathrm{~nm}$, respectively. The average fiber diameters of PET $6(887 \mathrm{~nm})$, PET $8(876 \mathrm{~nm})$ and PU(430nm) are listed in Table 1. The finer the fiber, the more flexible the fiber membrane. Therefore, the laminated fabric using the PU membrane will more flexible than the PET membrane.

Table I The basic properties of electrospun membranes

\begin{tabular}{llll} 
& PET6 & PET8 & PU \\
\hline Fiber diameter/nm & 887 & 876 & 430 \\
Thickness/ $\mu \mathrm{m}$ & 78.2 & 96.6 & 78.6 \\
Mass/g & 0.004 & 0.005 & 0.0048 \\
Porosity/\% & 87.27 & 87.12 & 83.04 \\
Water contact angle/ ${ }^{\circ}$ & $138.0 \pm 0.4$ & $138.0 \pm 0.5$ & $126.0 \pm 0.3$ \\
Surface density $/\left(\mathrm{g} \mathrm{m}^{-2}\right)$ & 13.3 & 16.7 & 16 \\
\hline
\end{tabular}

\section{Breathability test}

The results of the breathability test of the laminated fabrics with different electrospun nano fibrous membranes are shown in Table 2. As reference group, pristine electrospun nano fibrous membranes, that are PET 6, PET 8 and TPU, are also tested under the same condition. The air resistance values of PET 6, PET 8 and TPU are $3.9 \pm 0.6$, $5.8 \pm 0.3$ and $6.2 \pm 0.2$, respectively. This trend can be assigned to the fiber diameter and surface density, as shown in Table 1.

It can be seen that the air resistance value of the laminated fabric 
is obviously larger than that of the electrospun membrane. This means that the using of adhesive agent undermine the breath ability at some extent. Comparing with PU and PET8, both the thickness and porosity of PET8 are larger than them of PU, so the thickness and porosity of the nano fibrous membrane have an opposite influence on the air resistance value of the laminated fabric.

The electro spun nano fibrous membrane of the same material with different spinning times will also affect the air resistance value of the laminated fabric. From the performance of laminated fabric using PET membrane, the air resistance value [up to $72.3 \mathrm{kPa} /(\mathrm{s} \cdot \mathrm{m})]$ of the laminated fabric with membrane spinning $12 \mathrm{~h}$ was almost as high as triple times of that $[24.7 \mathrm{kPa} /(\mathrm{s} \cdot \mathrm{m})]$ of fabric with membrane spinning $4 \mathrm{~h}$. This indicates that the air resistance value of the laminated fabric increases as the thickness of the electros pun fiber. The air resistance value of the laminated fabric is also affected by the porosity of the electrospun nano fibrous membrane, as described before. The smaller the porosity of the membrane is, the greater the air resistance value of the laminated fabric is as it shown in Tables $1 \& 2$. Based on the study of laminated fabric with PET electrospun membrane or PU electrospun membrane, we prepared another mixed electrospun membrane, noted as PET/PU, using the similar electrospun conditions. It should be noted that the spinning time is $4 \mathrm{hrs}$ for PET and $4 \mathrm{hrs}$ for PU, respectively. It can be seen the air resistance value of laminated fabric with PET/PU is much lower than that of fabric PET 8.

\section{Moisture permeability}

Moisture permeability is vital to the hot and humid comfort of the clothing (especially in high temperature and high humidity environments). The moisture permeability of the laminated fabric with the electrospun nano fibrous membrane that acts as functional layer is shown in Table 2. Refer to the data listed in Table 1 and Table 3 , thickness, porosity and water absorption have a significant effect on the moisture permeability of the laminated fabric. In the high temperature condition, the polymer having a high water absorption capacity can improve the moisture permeability of the fabric. ${ }^{1}$ Therefore, the moisture permeability of the laminated fabric using the PU nano fibrous membrane as the functional layer is theoretically stronger than that of the PET membrane. The test results of water absorption capacity are listed in Table 3 .

Table 2 Test results of laminated fabrics with different electrospun nano fibrous membranes

\begin{tabular}{llllllll}
\hline Sample Property & PET 4 & PET 6 & PET 8 & PET I0 & PET I2 & PU & PET/PU \\
\hline Air resistance value $/(\mathrm{kPa} \cdot \mathrm{s} / \mathrm{m})$ & 24.7 & 37.5 & 45 & 61.3 & 72.3 & 20.1 & 6.2 \\
Moisture permeability $/\left[\mathrm{kg} /\left(\mathrm{m}^{2} \cdot \mathrm{d}\right)\right]$ & 9.45 & 8.93 & 8.11 & 7.39 & 7.12 & 9.33 & 8.82 \\
Hydrostatic pressure $/\left(\mathrm{cm} \mathrm{H}^{2} \mathrm{O}\right)$ & 16 & 23.8 & 25.1 & 25.8 & 26.4 & 27 & 36.1 \\
Warmth retention/\% & 22.78 & 23.42 & 24.05 & 25.95 & 25.95 & 21.52 & 22.12 \\
\hline
\end{tabular}

Table 3 Water absorption test results of nano fibrous membrane and laminated fabrics

\begin{tabular}{llll}
\hline Samples & $\mathbf{W}_{\mathbf{0}} / \mathbf{g}$ & $\mathbf{W}_{\mathbf{l}} / \mathbf{g}$ & Water absorption/\% \\
\hline PU nano fibrous membrane & $0.0052 \pm 0.0003$ & $0.0156 \pm 0.0002$ & $200 \pm 9.62$ \\
PET nano fibrous membrane & $0.0361 \pm 0.0002$ & $0.0249 \pm 0.0001$ & $44.97 \pm 0.83$ \\
Lining & $0.0233 \pm 0.0001$ & $0.0276 \pm 0.0001$ & $18.45 \pm 0.85$ \\
Fabric & $0.0491 \pm 0.0003$ & $0.0624 \pm 0.0002$ & $27.09 \pm 1.02$ \\
laminated fabric (PU) & $0.2207 \pm 0.0006$ & $0.02730 \pm 0.0008$ & $27.30 \pm 0.63$ \\
laminated fabric (PET I) & $1.0667 \pm 0.0010$ & $1.3270 \pm 0.0015$ & $24.40 \pm 0.23$
\end{tabular}

Since the PU nano fibrous membrane has strong hydrophilic groups (HNCOO) and hydrophilic segments, it has a very high water absorption (larger than 200\%), while the PET membrane has a relatively low water absorption (about 44.97\%). However, in case of the laminated fabric, the water absorption of the laminated fabrics using the PET electrospun membrane and the PU electrospun membrane were very similar, $24.40 \%$ and $23.70 \%$, respectively. This is because the weight percentage of nano fibrous membrane in laminated fabric is very low comparing with shell fabric and lining, the water absorption of the laminated fabric would be mainly affected by the shell fabric and lining. Although the thickness of PU is similar with that of PET 6 and the porosity is smaller than that of PET 6 as shown in Table 1, the moisture permeability of the laminated fabric with PU electrospun nano fibrous membrane is slightly higher than that of laminated fabric with PET 6, because of the better hydrophilicity. At the same time, the moisture permeability value of laminated fabric with PET/PU composite membrane is higher than that of the fabric with pristine PET 8 membrane as functional layer, indicating the hydrophilic groups and segments of PU is beneficial to the moisture permeability. Thus the effect of fibrous material on the moisture permeability of high porosity fabrics is significant.

Besides the water adsorption capacity, the thickness and porosity of electrospun nano fibrous membrane are important to the moisture permeability of electrospun membrane. According to the HagenPoises law, the moisture permeability of the laminated fabric is related to the thickness of the membrane, the porosity and the bending degree of the pores. The thickness of PET 8 electro spun membrane is less than that of PET 6 electro spun membrane, and microspores are more tortuous, so the moisture permeability of laminated fabric using PET 8 electrospun membrane is lower than that of laminated fabric with PET 6 electro spun membrane. In addition, the water vapor molecules are easily combined with the PU hydrophilic groups, so as to easily pass through the PU membrane, therefore, the laminated fabric with PU as a functional layer has a good moisture permeability of about $9.33 \mathrm{~kg} /\left(\mathrm{m}^{2} \bullet \mathrm{d}\right)$. 


\section{Water resistance}

Water resistance (expressed as a hydrostatic pressure) is an important parameter for waterproof fabrics. According to Laplace law (capillary waterproofing principle), the hydrostatic pressure value is proportional to the water contact angle and is inversely proportional to the pore size of the membrane. The thickness of membrane has also a great influence on the hydrostatic pressure of the laminated fabric. For the laminated fabric using PET electrospun membrane, the hydrostatic pressure value of laminated fabric increased with the increasing spinning time, in another word, thickness of electrospun membrane. Comparing with PET 6 membrane, the PU membrane has a similar thickness while lower porosity and higher surface density, thus the hydrostatic pressure of the laminated fabric with PU electrospun membrane as functional layer is higher. In case of PET/PU composite membrane, the hydrophobic property of PET and compact pore structure (low porosity and small pore size) lead to the

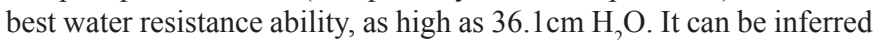
that the thicker membrane and the smaller pore size are, the greater the hydrostatic pressure is.
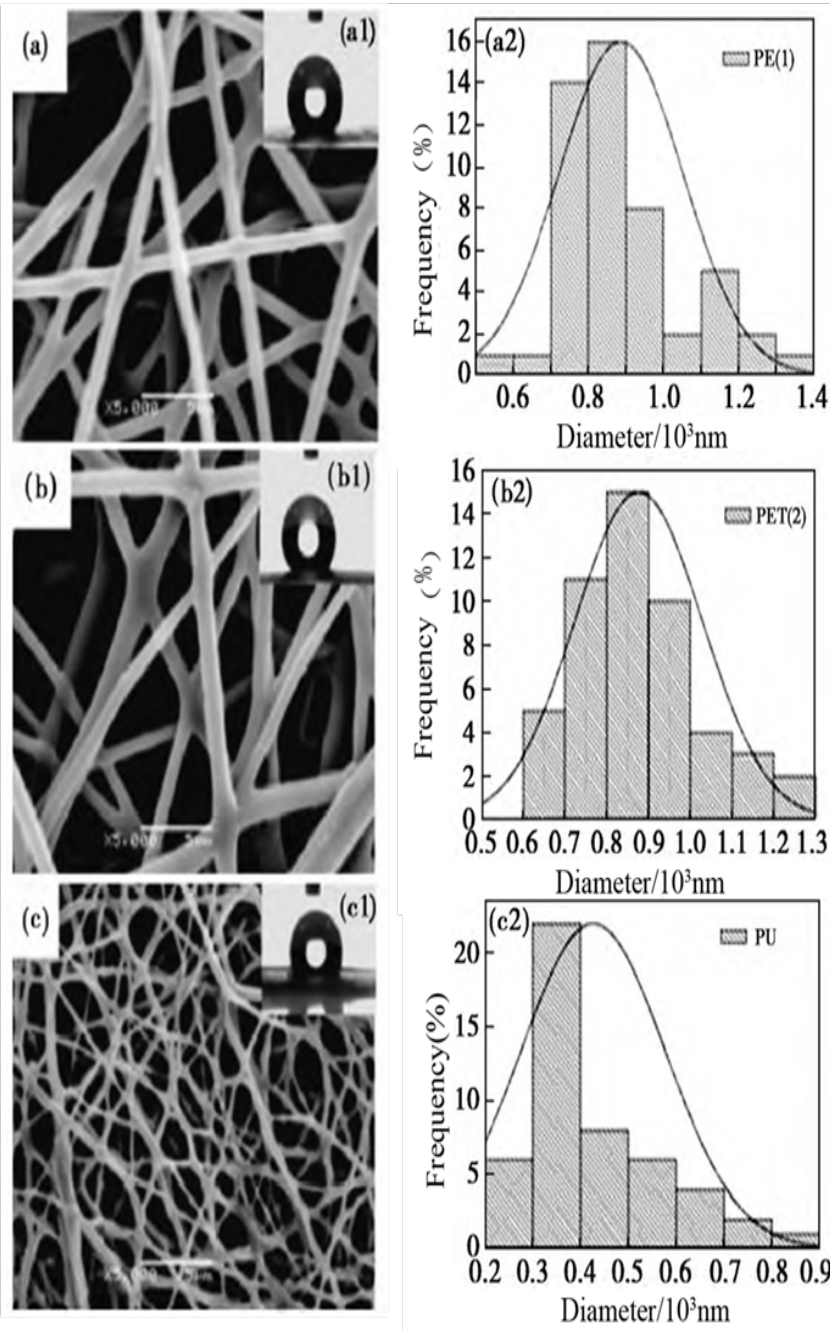

Figure 5 SEM pictures (left) and diameter distributions (right) of the electrospun membranes (a) PET6, (b) PET8 and (c) PU membrane. (al), $(\mathrm{bl})$ and $(\mathrm{cl})$ are the water contact angles of the corresponding membrane, respectively; (a2), (b2) and (c2) are the diameter distribution histograms of the corresponding membranes, respectively.

\section{Warmth retention}

It can be observed in Table 2 the warmth retention of the laminated fabrics with PET electro spun nano fibrous membrane as functional layer increases with the increasing spinning time and surface density, although the increment is not obvious. The warmth retention increases from $23.42 \%$ of PET 6 to $24.05 \%$ of PET 8 when the surface density increases from $13.3 \mathrm{~g} / \mathrm{m}^{2}$ to $16.7 \mathrm{~g} / \mathrm{m}^{2}$. And with the continuous lengthening the spinning time, that is thickening the electrospun nano fibrous membrane, the warmth retention of laminated fabric dost not boost further.

\section{Conclusion}

By directly spinning PET and PU nano fibrous membrane on hot melt adhesive and then hot pressing, it is a simpler method to obtain laminated fabric with much softer hand feeling than the traditional method.

The optimum hot pressing conditions for the laminated fabric using PET and PU electrospun nano fibrous membrane are determined by the peel strength. The optimum hot pressing conditions in this work (as the peel strength is maximum) are $130^{\circ} \mathrm{C}$ and $17.5 \mathrm{~s}$, respectively.

Hot pressing process can enhance the wind and water resistance of the fabric, but reduce its moisture permeability simultaneously. However, the laminated fabrics using the electrospun nano fibrous membrane as functional layer maintains good moisture permeability and water resistance property. In particular, the moisture permeability and water resistance property of the laminated fabric with PET/PU composite electrospun nano fibrous membrane as functional layer are $8.82 \mathrm{~kg} /\left(\mathrm{m}^{2} \cdot \mathrm{d}\right)$ and $36.1 \mathrm{~cm} \mathrm{H}_{2} \mathrm{O}$.

The waterproof and moisture permeability of the laminated fabric can be adjusted by changing the polymer type and thickness of the electrospun nano fibrous membrane, so that controlling the electro spinning process may be a feasible path to design waterproof and breathable laminate fabrics.

\section{Acknowledgments}

The authors would like to acknowledge the National Natural Science Foundation of China (NOs. 21274006, 51503005); Beijing Science and Technology Leading Talent Project (Z16111000490000); Importation and Development of High-Caliber Talents Project of Beijing Municipal Institutions - Beijing Great Wall Scholars Incubator Program (CIT\&TCD20150306); Beijing City Board of Education Upgrade Project (TJSHG201310012021); Beijing Institute of Clothing Innovation Team and Talent Selection and Training Program (PTTBIFT, 2014AL-68) and Key Research Project of Beijing Institute of Fashion Technology(2016A-03).

\section{Conflict of interest}

Author declares there is no conflict of interest in publishing the aticle.

\section{References}

1. Horrocks AR, Anand SC. Handbook of technical textiles. Cambridge, England: Woodhead Publishing Ltd; 2000. p. 282-315.

2. Quan H, Xing J, Fan Z. Synthesis and characterization of crystallized \&cross-linked high water vapor permeable polyurethane. J Textile Research. 2007;28(11):60-64. 
3. Hao RY, Zhu P. Can breathe functional fabric-laminated PTFE membrane. Advanced Textile Technology. 2007;15(1):52-54.

4. Yang FX, Wang LM. Laminated Composite Process Optimization. Shandong Textile Science \& Technology. 2012;53(1):47-49.

5.Zhou K. Preparation and characterization of polyamide reverse osmosis composite membrane based on poly (vinyl chloride) nano fibrous substrates. Dong Hua University, Shanghai, China; 2010.

6. Zheng YG, Wang J. Preparation of oxide nano fibers by electro spinning and their gas sensing properties. J Functional Materials. 2011;42(1):1-4.

7. Yang WJ, Fu J, HE Lei, et al. Preparation and characterization of chitosan/polycaprolactone vascular scaf folds by electro spinning. Acta Materiae Compositae Sinica. 2011;28(1):104-108.

8. Liu Y, Ma JG. Research status and development trend of waterproof and moisture permeable fabrics. Beijing Textile J. 2004;25(3):37-40.
9. Li M, Zhou JY. The research about the processing and performance of waterproof breathable laminated fabrics. Beijing Institute of Fashion Technology, Beijing, China; 2011.

10. Schreuder Gibson HL, Gibson P, Senecal K, et al. Protective Textile Materials Based on Electrospun Nano fibers. Chemical Fibers International. 2002;34(3):44-55.

11. Gibson P, Schreuder-Gibson H, Rivin D. Transport properties of porous membranes based on electrospun nano fibers. Colloids \& Surfaces a Physicochemical \& Engineering Aspects. 2001. p. 469-481.

12. Shen XY. Polymeric Materials Processing Principles. 2nd ed. Beijing: China Textile \& Apparel Press; 2009. p. 123. 